S. HAMADA

KODAI MATH. J.

$3(1980), 415-428$

\title{
ON THE CONDUCTORS OF $p$-CYCLIC KUMMER EXTENSIONS OF LOCAL NUMBER FIELDS
}

\author{
By Suguru Hamada
}

Introduction. Let $p$ be a prime number, $\mathbf{Q}_{p}$ be the rational $p$-adic number field, and $K$ be a finite extension over $\mathbf{Q}_{p}$ containing a primitive $p^{n}$-th root of unity.

An explicit formula of the norm residue symbol for the elements of $K$ is known (H. Hasse [3], M. Kneser [4], and I. R. Šafarevič [5]).

In this paper, using the explicit formula we describe the conductor of Kummer extension $K\left({ }^{n} \sqrt{A}\right) / K$ in some cases by means of the "exponents" of $A$ in its Šafarevič's representation (Theorem 1 and 2).

When $n=1$ the result is found in H. Hasse [1] (Remark 2). In $\S 1$, for convenience, we write down the outline of the Šafarevič's representation of the elements of $K$ and the explicit formula, following H. Hasse [3] and M. Kneser [4]. In $\S 2$, we give our theorems, in $\S 3$ we prove our theorems, and in $\S 4$ we give some remarks and examples.

\section{§1. Notations.}

$\mathbf{Z}$ : the ring of rational integers. $p:$ a prime number. $\mathbf{Q}_{p}:$ the rational $p$-adic number field. $\mathbf{Z}_{p}$ : the ring of integral elements of $\boldsymbol{Q}_{p} . \zeta_{n}$ : a primitive $p^{n}$-th root of unity. $K$ : a finite extension of $\mathbf{Q}_{p}$, containing $\zeta_{n}$. $K^{\times}$: the multiplicative group of non-zero elements of $K . \mathfrak{p}$ : the maximal ideal of $K . \quad \pi$ : a prime element of $K . \quad H_{m}$ : the multiplicative group $1+\mathfrak{p}^{m}(m=1,2, \cdots) . \operatorname{ord}^{\times}$: for a principal unit $\eta$ of $K$ we write $\operatorname{ord}^{\times}(\eta)=m$ if and only if $\eta \in H_{m}$ and $\eta \notin H_{m+1}$.

$\underset{p^{m}}{\sim}$ : for elements $A, B$ of $K^{\times}$we write $\underset{p^{m}}{\sim} B$ if and only if $A \in B K^{\times p^{m}}$. $\Omega$ : the group of $p^{n}$-primary numbers of $K . T$ : the inertia field of $K / \mathbf{Q}_{p} . \quad I$ : the ring of integral elements of $T . R$ : the multiplicative representatives of the residue class field of $K, R \subset I . R^{\times}: R^{\times}=R-\{0\}$. ord : the $p$-adic order function on $T$. $S_{p}$ : the trace mapping from $T$ to $\mathbf{Q}_{p}$.

$\bar{T}:$ the completion of the maximal unramified extension of $\boldsymbol{Q}_{p} . \quad \bar{I}:$ the ring of integral elements of $\bar{T} . \bar{R}$ : the multiplicative representatives of the residue class field of $\bar{T}, \bar{R} \subset \bar{I}$. $P$ : the Frobenius automorphism of the extension $T / \boldsymbol{Q}_{p}$. $\mathfrak{B}$ : the additive endomorphism of $\bar{I}$ defined by $\mathfrak{P}(\bar{\alpha})=\bar{\alpha}^{P}-\bar{\alpha}(\bar{\alpha} \in \bar{I}) . \quad e$ : the

Received September 18, 1979 
ramification index of the extension $K / \mathbf{Q}_{p} . \quad e_{m}$ : the ramification index of the extension $K / T\left(\zeta_{m}\right)$, where $\zeta_{m}=\zeta_{n}^{p^{n-m}}(1 \leqq m \leqq n)$. We have $e_{1}+e=e_{1} p, e_{1}=e_{m} p^{m-1}$.

$$
F: F=\left\{i \mid 1 \leqq i<e_{1} p,(i, p)=1\right\} .
$$

$\pi_{n}, \pi_{1}: \pi_{n}=1-\zeta_{n}, \pi_{1}=1-\zeta_{1}$. We have

$$
\pi_{n}^{p^{n}} \equiv-\pi_{n}^{p^{n-1}} p \equiv \pi_{1}^{p}=-\pi_{1} p \quad \bmod p^{e_{1} p+1} .
$$

$e_{0}, \kappa, \varepsilon_{0}, \varepsilon: e_{1}=e_{0} p^{\kappa-1}$ where $\left(e_{0}, p\right)=1(\kappa \geqq n)$,

and

$$
\pi^{e_{1} p} \equiv \varepsilon_{0}^{p^{\kappa}} \pi_{1}^{p} \quad \bmod p^{e_{1} p+1}\left(\varepsilon_{0} \in R^{\times}\right)
$$

$$
-p \equiv \varepsilon \pi^{e} \quad \bmod \mathfrak{p}^{e+1}\left(\varepsilon \in R^{\times}\right) .
$$

Now, for convenience, we write down the outline of Šafarevič's representation of elements of $K$ following $H$. Hasse [3]. Generally, if a system $S=\left\{\eta_{k}(\gamma) \mid \gamma \in R, k=1,2, \cdots\right\}$ is given so that $\eta_{k}(\gamma) \equiv 1-\gamma \pi^{k} \bmod \mathfrak{p}^{k+1}$, then every element $\eta \in H_{1}$ is written uniquely as follows:

$$
\eta=\prod_{k=1}^{\infty} \eta_{k}(\gamma), \quad \eta_{k}(\gamma) \in S
$$

Such a system $S$ is given by Šafarevič's $E$-function and $E^{*}$-function. The definitions and some properties of these functions are as follows. We define:

$$
E(\alpha, x)=\prod_{\substack{m=1 \\(m, p)=1}}^{\infty}\left(1-\alpha^{m} x^{m}\right)^{\mu(m) / m}, \quad \text { where } \quad \alpha \in R, x \in \mathfrak{p}
$$

and $\mu$ is the Möbius function.

$$
E(\alpha, x)=\sum_{\nu=0}^{\infty} E\left(\alpha_{\nu}, x\right)^{p}, \quad \text { where } \alpha=\sum \alpha_{\nu} p^{\nu} \in I \quad\left(\alpha_{\nu} \in R\right) .
$$

Then

$$
E(\alpha, x) \equiv 1-\alpha x \bmod x^{2}
$$

and

$$
\begin{aligned}
& E(\alpha+\beta, x)=E(\alpha, x) \cdot E(\beta, x) \\
& E(a \alpha, x)=E(\alpha, x)^{a} \quad \text { where } \alpha, \beta \in I \text { and } a \in \mathbf{Z}_{p}
\end{aligned}
$$

Next, for $\alpha \in I$ we define

$$
E^{*}(\alpha)=E\left(p^{n} \bar{\alpha}, \tilde{\pi}_{n}\right)=E\left(\bar{\alpha}, \tilde{\pi}_{n}\right)^{p^{n}}
$$

where $\mathfrak{P}(\bar{\alpha})=\alpha(\bar{\alpha} \in I), \zeta_{n}=E\left(1, \tilde{\pi}_{n}\right)$ and $E\left(\bar{\alpha}, \tilde{\pi}_{n}\right)$ is defined by the same formula as before. Then

$$
E^{*}(\alpha) \equiv 1-\alpha^{p^{n-1}} \pi_{1}^{p} \quad \bmod \mathfrak{p}^{e_{1} p+1}(\alpha \in R) \text { and }
$$




$$
\begin{aligned}
& E^{*}(\alpha+\beta)=E^{*}(\alpha) E^{*}(\beta) \\
& E^{*}(a \alpha)=E^{*}(\alpha)^{a} \quad \text { where } \quad \alpha, \beta \in I \quad \text { and } \quad a \in \mathbf{Z}_{p} .
\end{aligned}
$$

Moreover, $\left\{E^{*}(\alpha) \mid \alpha \in I\right\} \cdot K^{\times p^{n}}=\Omega$.

The following congruences are well known (H. Hasse [2]). For an integral element $\alpha$ of $K$, let $\eta \equiv 1-\alpha \pi^{2} \bmod \mathfrak{p}^{2+1}$ then

$$
\eta^{p} \equiv\left\{\begin{array}{lll}
1-\alpha^{p} \pi^{\imath p} & \bmod \mathfrak{p}^{2 p+1} & \text { if } i<e_{1} \\
1-\left(\alpha^{p}-\varepsilon \alpha\right) \pi^{e_{1} p} & \bmod \mathfrak{p}^{e_{1} p+1} & \text { if } i=e_{1} \\
1-\alpha p \pi^{2} & \bmod \mathfrak{p}^{2+e+1} & \text { if } i>e_{1} .
\end{array}\right.
$$

Now, as in Notations, let $F=\left\{i \mid 1 \leqq i<e_{1} p,(i, p)=1\right\}$ then the $e$ integers $k\left(e_{1}<k \leqq e_{1} p\right)$ are written uniquely

$$
k=i p^{\kappa_{i}}\left(i \in F, \kappa_{i} \geqq 0, \kappa_{e_{0}}=\kappa\right)
$$

and every positive integer $k$ is written uniquely as follows:

$$
\begin{aligned}
& \text { if } k \leqq e_{1} \text { then } k=i p^{\nu_{i}}\left(i \in F, 0 \leqq \nu_{i}<\kappa_{\imath}\right) \\
& \text { if } k>e_{1} \text { then } k=i p^{\kappa_{i}}+\nu_{i}^{\prime} e\left(i \in F, \nu_{i}^{\prime} \geqq 0\right) .
\end{aligned}
$$

From (2) and (4) we have

$$
\begin{aligned}
E\left(\alpha p^{\nu}, \pi^{i}\right) & =E\left(\alpha, \pi^{i}\right)^{p^{\nu} \imath} \equiv\left(1-\alpha \pi^{i}\right)^{p^{\nu} \imath} \\
& \equiv 1-\alpha^{p^{\nu} \imath} \pi^{k} \quad \bmod \mathfrak{p}^{k+1}
\end{aligned}
$$

$\left(\alpha \in R, 1 \leqq k \leqq e_{1}, k=i p^{\nu} i\right)$.

The above congruences hold also for $\nu_{i}=\kappa_{\imath}$ if $i \neq e_{0}$ (i. e. $e_{1}<k<e_{1} p$, $\left.k=i p^{\kappa_{i}}\right)$. And

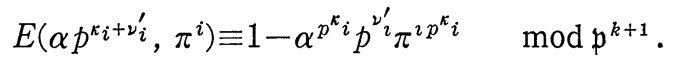

$\left(\alpha \in R, e_{1} p<k, k=i p^{\kappa_{i}}+\nu_{i}^{\prime} e\left(i \neq e_{0}\right), \nu_{i}^{\prime}>0\right)$. For the exceptional $k=e_{1} p+\nu^{\prime} e\left(\nu^{\prime} \geqq 0\right)$ corresponding to $i=e_{0}$, we have from (3) and (4)

$$
E^{*}\left(\alpha p^{\nu^{\prime}}\right)=E^{*}(\alpha)^{p^{\nu^{\prime}}} \equiv\left(1-\alpha^{p^{n-1}} \pi_{1}^{p}\right)^{p^{\nu^{\prime}}} \equiv 1-\alpha^{p^{n-1}} p^{\nu^{\prime}} \pi_{1}^{p}
$$

$\bmod \mathfrak{p}^{k+1}(\alpha \in R)$.

Since $R^{p^{m}}=R(m \geqq 1)$, a desired system $S$ has been given and from (1) every $\eta \in H_{1}$ is represented by $E$-function and $E^{*}$-function. Consequently every element $A \in K^{\times}$is represented uniquely as follows :

(̌́) $\quad A=\pi^{a} \rho \prod_{i \in F} E\left(\alpha_{\imath}, \pi^{i}\right) E^{*}(\alpha)\left(a \in \mathbf{Z}, \rho \in R^{\times}, \alpha_{\imath}, \alpha \in I \quad \alpha_{e_{0}}: \bmod p^{\kappa}\right.$ reduced. $)$

Now, for every $m(1 \leqq m \leqq n)$, we have 


$$
\pi^{a} \rho \prod_{i \in F} E\left(\alpha_{\imath}, \pi^{i}\right) E^{*}(\alpha) \underset{p^{m}}{\sim} \pi^{a^{\prime}} \rho^{\prime} \prod_{i \in F} E\left(\alpha_{\imath}^{\prime}, \pi^{i}\right) E\left(\alpha^{\prime}\right)
$$

if and only if $a \equiv a^{\prime} \bmod p^{m}, \alpha_{i} \equiv \alpha_{\imath}^{\prime} \bmod p^{m}(i \in F)$, and $\alpha \equiv \alpha^{\prime} \bmod p^{m}, \mathfrak{B}$ where the last congruence means that there exist $\delta, \theta \in I$ such that $\alpha-\alpha^{\prime}=p^{m} \delta+\mathfrak{P}(\theta)$.

In the following we write $\prod_{i}$ instead of $\prod_{i \in F}$ and $\sim$ instead of $\underset{p^{n}}{\sim}$.

[EXPLICIT FORMULA] (H. Hasse [3], M. Kneser [4] and I. R. Šafarevič [5]) Let $\mathrm{A}, \mathrm{B}$ be two elements of $K^{\times}$such that

$$
A \sim \pi^{a} \prod_{i} \mathrm{E}\left(\alpha_{\imath}, \pi^{i}\right) \mathrm{E}^{*}(\alpha), \quad B \sim \pi^{b} \prod_{j} \mathrm{E}\left(\beta, \pi^{j}\right) \mathrm{E}^{*}(\beta)
$$

then the norm residue symbol $(A, B)$ is given by

(9) If $p \neq 2 \quad(A, B)=\zeta_{n}^{\mathrm{Sp}(a \beta-b \alpha+\gamma)}$

where $\prod_{\imath, j \in F} \mathrm{E}\left(j \alpha_{i} \beta_{\jmath}, \pi^{\imath+j}\right) \sim \prod_{k} \mathrm{E}\left(\gamma_{k}, \pi^{k}\right) \mathrm{E}^{*}(\gamma)$.

(10) If $p=2 \quad(A, B)=\zeta_{n}^{\mathrm{Sp}}(a \beta-b \alpha+\gamma)$

where

$$
\begin{array}{r}
(-1)^{a b} \prod_{\imath, j \in F}^{\infty}\left[\mathrm{E}\left(j \alpha_{i} \beta_{j}, \pi^{\imath+j}\right) \prod_{\mu, \nu \geq 1}^{\infty} \mathrm{E}\left(\left(2^{\mu-1}+j 2^{\nu-1}\right) \alpha_{\imath}^{P^{\mu}} \beta_{J}^{P^{\nu}}, \pi^{\left.2^{\mu_{\imath+2} 2^{\nu}}\right)}\right]\right. \\
\left.\sim \prod_{k} \mathrm{E} \gamma_{k}, \pi^{k}\right) \mathrm{E}^{*}(\gamma)
\end{array}
$$

\section{§. Theorems.}

We write also $\pi^{a} \prod_{i} E\left(\alpha_{\imath}, \pi^{i}\right) E^{*}(\alpha)=\left\langle\alpha_{0}, \alpha_{1}, \cdots, \alpha\right\rangle$ where $\alpha_{0}=a$.

The aim of this paper is to describe, in some cases, the conductor $\mathfrak{p}^{f}$ of the extension $K\left(\sqrt[p^{n}]{A}\right) / K$ by means of conditions on $\alpha_{0}, \alpha_{\imath}(i \in F)$.

From the facts in $\S 1$, the extension $K\left(\sqrt[p^{n}]{A}\right) / K$ is unramified if and only if $\alpha_{i} \equiv 0 \bmod p^{n}$ for all $i \in F$ and $i=0$.

Thus we consider only the case when for some $r(1 \leqq r \leqq n)$ there exists $i$ $\left(i=0\right.$ or $i \in F$ ) such that $\alpha_{i} \neq 0 \bmod p^{r}$. And we denote $i_{r}$ the least suffix $i$ for which $\alpha_{i} \neq 0 \bmod p^{r}$. If $i_{r}$ exists then $i_{r+1}, \cdots, i_{n}$ exist and

$$
e_{1} p-1 \geqq i_{r} \geqq \cdots \geqq i_{n-1} \geqq i_{n} \geqq 0 \text {. }
$$

When $i_{r}$ exists we set $f_{r}=e_{1} p+(n-r) e-i_{r}+1$.

Moreover, for convenience, we set $i_{n+1}=i_{n}$ and $f_{n+1}=e_{1} p-e-i_{n+1}+1$. Then $f_{n}>f_{n+1}$ holds. This definition is natural in the following sense; if $i_{n+1}$ is the least suffix $i$ for which $\alpha_{i} \neq 0 \bmod p^{n+1}$, we have $i_{n+1} \leqq i_{n}$; here if $i_{n+1}<i_{n}$ we can take $B=\left\langle 0, \cdots, 0^{i_{n+1}}, \cdots, \alpha_{\imath_{n}}, \cdots\right\rangle$ instead of $A$; for this $B$ we have $i_{n+1}=i_{n}$.

Now, it follows from $\S 1$ that the extension $K\left(\sqrt[n^{n}]{A}\right) / K$ is a totally ramified 
extension of degree $p^{n}$ if and only if $i_{1}$ exists.

THEOREM 1. The extension $K\left(\sqrt[p^{n}]{A}\right) / K$ is a totally ramified extension of degree $p^{n}$ if and only if there exists $i(\imath=0$ or $\imath \in F)$ such that $\alpha_{i} \neq 0 \bmod p$. And, then

$$
f \leqq \operatorname{Max}\left\{f_{1}, f_{2}\right\}
$$

where $\mathfrak{p}^{f}$ is the conductor of the extension $K\left(\sqrt[p^{n}]{A}\right) / K$.

Moreover, $f=\operatorname{Max}\left\{f_{1}, f_{2}\right\}$ holds if and only if $e+\imath_{2} \neq \imath_{1}$ (..e. $f_{2} \neq f_{1}$ ) or $\alpha_{\imath_{2}} \varepsilon \neq \alpha_{\imath_{1}} p \bmod p^{2}$, where $-p \equiv \varepsilon \pi^{e} \bmod \mathfrak{p}^{e+1}\left(\varepsilon \in R^{\times}\right)$.

Remark. By the above remark, in the case $n=1$, our Theorem asserts that $f=f_{1}$. Moreover, for $n \geqq 2, e+i_{1}=i_{2}$ and $\alpha_{\imath_{2}} \varepsilon \equiv \alpha_{\imath_{1}} p \bmod p^{2}$ occures in these cases when $p \neq 2$ or $p=2$ and $T \supseteqq Q_{2}$. For example, in these cases, let $1 \leqq i_{2}<e_{1}$, $e+i_{2}=i_{1}$ and $A \sim E\left(\gamma p, \pi^{\imath_{2}}\right) E\left(1, \pi^{i_{1}}\right)$ where $\gamma \varepsilon=1\left(\gamma \in R^{\times}\right)$.

Now, THEOREM 1 can be generalized easily to the case when $K\left(\sqrt[p^{n}]{A}\right) / K$ contains an unramified subfield:

THEOREM 2. For integer $m(1 \leqq m \leqq n)$, if $\alpha_{i} \equiv 0 \bmod p^{m-1}$ for all $i \in F$ and $i=0$ and there exists some $i(i \in F$ or $i=0)$ such that $\alpha_{i} \neq 0 \bmod p^{m}$, then

$$
f \leqq \operatorname{Max}\left\{f_{m}, f_{m+1}\right\}
$$

where $\mathfrak{p}^{f}$ is the conductor of the extension $K\left(\sqrt[n^{n}]{A}\right) / K$. Moreover, $f=\operatorname{Max}\left\{f_{m}\right.$, $\left.f_{m+1}\right\}$ holds if and only if $e+i_{m+1} \neq \imath_{m}$ (i.e. $f_{m+1} \neq f_{m}$ ) or $\alpha_{\imath_{m+1}} \varepsilon \neq \alpha_{\imath_{m}} p \bmod p^{m+1}$, where $-p \equiv \varepsilon \pi^{e} \bmod \mathfrak{p}^{e+1}\left(\varepsilon \in R^{\times}\right)$.

Remark. In the case $m=n$, our Theorem asserts that $f=f_{n}$. In fact, Theorem 2 is proved by Theorem 1 as follows: By assumption,

$$
\alpha_{0}=\alpha_{0}^{\prime} p^{m-1} \text { and } \alpha_{\imath}=\alpha_{\imath}^{\prime} p^{m-1}(i \in F) \text { for some } \alpha_{0}^{\prime} \in Z \text { and } \alpha_{i}^{\prime} \in I \text {. }
$$

So we have $A_{p^{m-1}} E^{*}(\alpha)$ and $L=K\left(\sqrt[p^{m-1}]{A}\right)=K\left(\sqrt[p^{m-1}]{E^{*}(\alpha)}\right)$ is unramified over $K$.

Let $B=\sqrt[p^{m-1}]{A}$ then $K\left(\sqrt[p^{n}]{A}\right)=K\left(\sqrt[p^{n-m+1}]{B}\right)$ and $B \overbrace{p^{n-m+1}}\left\langle\alpha_{0}^{\prime}, \alpha_{1}^{\prime}, \cdots, \gamma\right\rangle$ in $L$ where $\gamma$ is an integral element of the inertia field of $L / \boldsymbol{Q}_{p}$.

Now, the least suffix such that $\alpha_{1}^{\prime} \neq 0 \bmod p$ is $i_{m}$. Applying Theorem 1 to the totally ramified extension $K\left(\sqrt[p^{n-m+1}]{B^{-}}\right) / L$ we have $f \leqq \operatorname{Max}\left\{f_{m}, f_{m+1}\right\}$, where

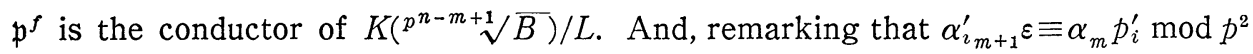
is equivalent to $\alpha_{\imath_{m+1}} \varepsilon \equiv \alpha_{i_{m}} p \bmod p^{m+1}$ we have also the necessary and sufficient conditions for $f=\operatorname{Max}\left\{f_{m}, f_{m+1}\right\}$. Since $L / K$ is unramified, as for the conductor of $K\left(\sqrt[p^{n}]{A}\right) / K$ we have Theorem 2 . 


\section{$\S 3$. Proof of Theorem 1 .}

Now, for the proof of Theorem 1, we prove some Lemmas. In the proofs we use following facts.

For a principal unit $B$ in $K$ and positive integer $r$,

$$
\text { if } B \equiv 1 \bmod \mathfrak{p}^{e_{1} p+(r-1) e+1} \text { then } B \underset{p^{r}}{\sim} 1 .
$$

(J. P. Serre [5], p. 219, Proposition 9).

By (5), (6), and (7)

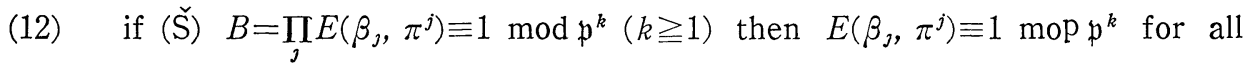
$j \in F$ and $E^{*}(\beta) \equiv 1 \bmod \mathfrak{p}^{k}$. By (2) and (4)

(13) if $s>e_{1}$ then $\operatorname{ord}^{\times} E\left(\alpha p^{m}, \pi^{s}\right)=s+m e(\alpha \in I, \alpha \neq 0 \bmod p, m \geqq 0$ : integer).

(14) if $i<j\left(i, j \in F, i \neq e_{0}, j \neq e_{0}\right)$

$\operatorname{ord}^{\times} E\left(p^{m}, \pi^{i}\right)<\operatorname{ord}^{\times} E\left(p^{m}, \pi^{j}\right)$ and when $m \leqq \kappa-1$ (especially when $m \leqq n-1$ ) this inequality holds also for $i=e_{0}$ or $j=e_{0}$.

In fact, let $i \neq e_{0}$ and $j \neq e_{0}$, since $\imath<j$ we have $\kappa_{i} \geqq \kappa_{\jmath}$, if $\kappa_{\imath}=\kappa_{3}$ then the result follows immediately, so let $\kappa_{\imath}>\kappa_{j}$. If $m \leqq \kappa_{j}<\kappa_{\imath}$ then $\operatorname{ord}^{\times} E\left(p^{m}, \pi^{i}\right)$ $=i p^{m}<j p^{m}=\operatorname{ord}^{\times} E\left(p^{m}, \pi^{j}\right)$, if $\kappa_{j}<m \leqq \kappa_{\imath}$ then $\operatorname{ord}^{\times} E\left(p^{m}, \pi^{j}\right)-\operatorname{ord}^{\times} E\left(p^{m}, \pi^{i}\right)=j p^{\kappa_{j}}$ $+\left(m-\kappa_{j}\right) e-i p^{m}>0$, because $j p^{\kappa_{j}}-i p^{m}>e_{1}-e_{1} p=-e,\left(m-\kappa_{j}\right) e \geqq e$, and if $\kappa_{j}<\kappa_{i}<m$ then $\operatorname{ord}^{\times} E\left(p^{m}, \pi^{j}\right)-\operatorname{ord}^{\times} E\left(p^{m}, \pi^{i}\right)=j p^{\kappa_{j}}-i p^{k_{i}}+\left(\kappa_{i}-\kappa_{j}\right) e>0$, because $j p^{\kappa_{j}}-i p^{\kappa_{i}}>$ $-e$ and $\left(\kappa_{i}-\kappa_{j}\right) \geqq e$. Furthermore, if $m \leqq \kappa-1$ then, since $\operatorname{ord}^{\times} E\left(p^{m}, \pi^{e_{0}}\right)=e_{0} p^{m}$, the inequality holds also for $i=e_{0}$ or $j=e_{0}$.

LEMMA 1. Let $n \geqq 1$, for a given integer $t(t=0$ or $t \in F)$, let $k=e_{1} p+(n-1) e$ $-t+1$ and

$$
B=\prod_{\jmath} E\left(\beta_{\jmath}, \pi^{j}\right) E^{*}(\beta) \equiv 1 \quad \bmod \mathfrak{p}^{k}
$$

Then, (i) when $t=0, \beta, \equiv 0 \bmod p^{n}$ for all $\jmath \in F$ and $\beta \equiv 0 \bmod p^{n}$, $\mathfrak{P}$.

(ii) When $1 \leqq t<e, \beta_{j} \equiv 0 \bmod p^{n-1}$ for all $\jmath \in F$ and $\beta \equiv 0 \bmod p^{n-1}, \mathfrak{P}$ and moreover $\beta_{j} \equiv 0 \bmod p^{n}$ if $j \leqq e_{1} p-t$.

(iii) When $e<t<e_{1} p, \quad \beta_{j} \equiv 0 \bmod p^{n-2}$ for all $j \in F, \beta \equiv 0 \bmod p^{n-2}, \mathfrak{B}$ and moreover

$$
\beta_{j} \equiv \begin{cases}0 \bmod p^{n-1} & \text { if } j \leqq e_{1} p+e-t \\ 0 \bmod p^{n} & \text { if } j \leqq e_{1} p-t .\end{cases}
$$

Remark. For $n=1$, the parts of $\bmod p^{n-1}$ and $p^{n-2}$ in the Lemma 1 and its proof may be omitted.

Proof. (i) follows immediately from (11) and (8).

(ii) Since $t<e$ we have $k>e_{1} p+(n-2) e+1$ and $B \underset{p^{n-1}}{ } 1$ by (11) and so by (8), $\beta_{j} \equiv 0 \bmod p^{n-1}$ for all $j \in F$ and $\beta \equiv 0 \bmod p^{n-1}, \mathfrak{P}$.

Next we show that $\beta_{\jmath} \equiv 0 \bmod p^{n}$ if $j \leqq e_{1} p-t$. For, let $\beta$, $\neq 0 \bmod p^{n}$ for 
some $j, j \leqq e_{1} p-t$ then since $e_{1} p-t>e_{1}$ we have $\operatorname{ord}^{\times} E\left(\beta_{\jmath}, \pi^{j}\right) \leqq \operatorname{ord}^{\times} E\left(p^{n-1}, \pi^{e_{1} p-t}\right)$ $=e_{1} p-t+(n-1) e<k$ by (13) and (14), this contradicts to the assumption $E\left(\beta_{j}, \pi^{j}\right) \equiv 1 \bmod \mathfrak{p}^{k}$.

(iii) Since $t<e_{1} p \leqq 2 e$ we have $k>e_{1} p+(n-3) e+1$. It follows that $B \overbrace{p^{n-2}} 1$ and $\beta_{j} \equiv 0 \bmod p^{n-2}$ for all $j \in F, \beta \equiv 0 \bmod p^{n-2}, \mathfrak{P}$. Next we show that $\beta_{j} \equiv 0$ $\bmod p^{n-1}$ if $j \leqq e_{1} p+e-t$. Let $\beta, \neq 0 \bmod p^{n-1}$ for some $j, \jmath \leqq e_{1} p+e-t$, then

$$
\operatorname{ord}^{\times} E\left(\beta_{\jmath}, \pi^{j}\right) \leqq \operatorname{ord}^{\times} E\left(p^{n-2}, \pi^{e_{1} p+e-t}\right)=e_{1} p+e-t+(n-2) e<k,
$$

by (13) and (14) but this contradicts to our assumption.

Finally we show that $\beta_{j} \equiv 0 \bmod p^{n}$ if $j \leqq e_{1} p-t$. Let $\beta_{j} \neq 00 \bmod p^{n}$ for some $j, j \leqq e_{1} p-t$, then $\operatorname{ord}^{\times} E\left(\beta_{\jmath}, \pi^{j}\right) \leqq \operatorname{ord}^{\times} E\left(p^{n-1}, \pi^{i}\right)$ where $\imath=e_{1} p-t$. We show that $\operatorname{ord}^{\times} E\left(p^{n-1}, \pi^{i}\right)=m<k$ then the proof is completed.

Since $i<e_{1}$ it follows that $\kappa_{i} \geqq 1$. Now, in the case $\kappa_{\imath} \leqq n-1$, we have

$$
k-m=i+(n-1) e+1-\left(i p^{\kappa_{i}}+\left(n-1-\kappa_{\imath}\right) e\right)=\imath-i p^{\kappa_{i}}+\kappa_{i} e+1
$$

by (6). If $\kappa_{2}=1$ then

$$
k-m=-i(p-1)+e+1>-e_{1}(p-1)+e+1>0,
$$

if $\kappa_{i} \geqq 2$ then $k-m>0$ because $i p^{\kappa_{i}} \leqq e_{1} p \leqq 2 e$.

And in the case $\kappa_{\imath}>n-1$, we have $m=i p^{n-1} \leqq e_{1}$ by (5), and $k-m=i+(n-1) e$ $+1-i p^{n-1}$. If $n=1$ then clearly $k-m>0$ and if $n \geqq 2$ we have $k-m>0$ since $i p^{n-1} \leqq i p^{\kappa_{i}-1} \leqq e_{1} \leqq e$.

Q.E.D.

Proof of Theorem 1 in the case $p \neq 2$.

In the following, when the conductor of $K\left(\sqrt[p^{n}]{A}\right) / K$ is $\mathfrak{p}^{f}$ we write $f=f(A)$.

LEMMA 2. Let $n \geqq 1$ and $p \neq 2$ then

$$
\text { if } A \sim \pi^{a}(a \in \boldsymbol{Z}, a \neq 0 \bmod p) \text {, }
$$

$$
f(A)=e_{1} p+(n-1) e+1,
$$

(ii) If $A \sim E\left(\alpha_{\imath}, \pi^{i}\right)\left(i \in F, \alpha_{i} \in I, \alpha_{i} \neq \equiv \bmod p\right)$,

$$
f(A)=e_{1} p+(n-1) e-i+1 .
$$

Proof. (i) Let $B \equiv 1 \bmod \mathfrak{p}^{e_{1} p+(n-1) e+1}$ then $B \sim 1$ by (11) so we have $(A, B)$ $=1$ and $f(A) \leqq e_{1} p+(n-1) e+1$. Next, let $B=E^{*}\left(\delta p^{n-1}\right)$ where $\delta \in R^{\times}$and $\operatorname{Sp}(\delta) \equiv 1$ $\bmod p$. Then $B \equiv 1 \bmod p^{e_{1} p+(n-1) e}$ by $(7)$ and $(A, B)=\zeta_{n}^{\mathrm{Sp}\left(a \delta p^{n-1}\right.} \neq 1$. So we have

$$
f(A) \leqq e_{1} p+(n-1) e+1 .
$$

(ii) Proof of $f(A) \leqq e_{1} p+(n-1) e-i+1$. Let

$$
(\check{\mathrm{S}}) \quad B=\prod_{\jmath} E\left(\beta_{\jmath}, \pi^{j}\right) E^{*}(\beta) \equiv 1 \quad \bmod \mathfrak{p}^{e_{1} p+(n-1) e-\imath+1}
$$


We show that $E\left(j \alpha_{i} \beta_{\jmath}, \pi^{\imath+j}\right) \sim 1$ for all $\jmath \in F$ by showing that $\alpha_{i} \beta_{\jmath} \equiv 0 \bmod p^{n}$ or $\operatorname{ord}^{\times} E\left(j \alpha_{\imath} \beta_{\jmath}, \pi^{\imath+j}\right)>e_{1} p+(n-1) e$. Then we have the result by the explicit formula (9).

Case $1 ; 1 \leqq i<e$. By Lemma 1 , if $\jmath \leqq e_{1} p-\imath$ then $\beta_{j} \equiv 0 \bmod p^{n}$ so we have $\alpha_{i} \beta_{j} \equiv 0 \bmod p^{n}$. If $j>e_{1} p-\imath$ then $\jmath>e_{1}$ and $\beta_{j} \equiv 0 \bmod p^{n-1}$ by Lemma 1 so we have $\operatorname{ord}^{\times} E\left(\jmath \alpha_{i} \beta_{\jmath}, \pi^{\imath+j}\right) \geqq \imath+\jmath+(n-1) e>e_{1} p+(n-1) e$ by (13).

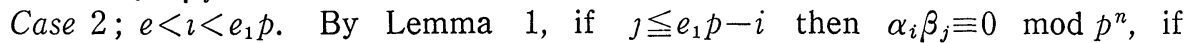
$e_{1} p-i<\jmath \leqq e_{1} p+e-\imath$ then $\alpha_{i} \beta_{j} \equiv 0 \bmod p^{n-1}$ and so $\operatorname{ord}^{\times} E\left(j \alpha_{i} \beta_{\jmath}, \pi^{2+j}\right)>e_{1} p+(n-1) e$ by (13), and if $e_{1} p+e-i<j$ then $\alpha_{i} \beta_{j} \equiv 0 \bmod p^{n-2}$ and $\operatorname{ord}^{\times} E\left(j \alpha_{i} \beta_{j}, \pi^{2+j}\right)>e_{1} p$ $+e+(n-2) e=e_{1} p+(n-1) e$ by $(13)$.

Proof of $f(A) \geqq e_{1} p+(n-1) e-i+1$. It is enough to show that there exists $B$ such tnat

$$
B \equiv 1 \bmod \mathfrak{p}^{e_{1} p+(n-1) e-\imath} \quad \text { and }(A, B) \neq 1 .
$$

Case $1 ; 1 \leqq \imath<e . \quad$ Let $B=E\left(\beta_{\jmath}, \pi^{j}\right)$ where $j=e_{1} p-\imath\left(\jmath \in F, j>e_{1}\right)$ and $\beta_{\jmath}=\delta p^{n-1}$ $\left(\delta \in R^{\times}\right.$will be determined below). Then $E\left(\beta_{\jmath}, \pi^{j}\right) \equiv 1 \bmod \mathfrak{p}^{e_{1} p-\imath+(n-1) e}$ by (13), and $E\left(j \alpha_{i} \beta_{\jmath}, \pi^{\imath+j}\right) \equiv 1-\jmath \alpha_{i} \delta p^{n-1} \pi^{e_{1} p} \equiv 1-\delta_{0} \delta p^{n-1} \varepsilon_{0}^{p^{\kappa}} \pi_{1}^{p} \bmod p^{e_{1} p+(n-1) e+1}$ where $j \alpha_{2}$ $\equiv \delta_{0} \bmod p\left(\delta_{0} \in R^{\times}\right)$and $\varepsilon_{0}$ is that of Notations. On the other hand, by (7) $E^{*}\left(\left(\delta_{0} \delta \varepsilon_{0}^{p^{\kappa}}\right)^{p^{-(n-1)}} p^{n-1}\right) \equiv 1-\delta_{0} \delta \varepsilon_{0}^{p^{\kappa}} p^{n-1} \pi_{1}^{p} \bmod p^{e_{1} p+(n-1) e+1}$. So, we have $E\left(\jmath \alpha_{\imath} \beta_{\jmath}, \pi^{\imath+j}\right)$ $\sim E^{*}\left(\left(\delta_{0} \delta \varepsilon_{0}^{p \kappa}\right)^{p-(n-1)} p^{n-1}\right)$ and in explicit formula (9), we have $\gamma=\left(\delta_{0} \delta \varepsilon_{0}^{p^{\kappa}}\right)^{p-(n-1)} p^{n-1}$. Now, if we choose $\delta$ so that $\operatorname{Sp}\left(\left(\delta_{0} \delta \varepsilon_{0}^{p^{\kappa}}\right)^{p^{-(n-1)}}\right) \equiv 1 \bmod p$ then $B \equiv 1 \bmod \mathfrak{p}^{e_{1} p+(n-1) e-\imath}$ and $(A, B)=\zeta_{n} \mathrm{Sp}(r)=\zeta_{n}^{p-1} \neq 1$.

Case $2 ; i>e$. Let $B=E\left(\beta_{\jmath}, \pi^{j}\right)$ where $\jmath=e_{1} p+e-i\left(\jmath \in F\right.$ and $\left.j>e_{1}\right)$ and $\beta_{j}=\delta p^{n-2} \quad\left(\delta \in R^{\times}\right.$will be determied below). Then we have $E\left(\jmath \alpha_{i} \beta_{\jmath}, \pi^{\imath+j}\right)$ $\equiv 1-j \alpha_{i} \delta p^{n-2} \pi^{e_{1} p+e} \equiv 1+j \alpha_{i} \delta \varepsilon^{-1} p^{n-1} \pi^{e_{1} p} \equiv 1-\delta_{0} \delta \varepsilon_{0}^{p^{\kappa}} p^{n-1} \pi_{1}^{p} \bmod p^{e_{1} p+(n-1) e+1}$ where $-j \alpha_{i} \varepsilon^{-1} \equiv \delta_{0} \bmod p\left(\delta_{0} \in R^{\times}\right)$and $\varepsilon$ is that of Notations. Thus, just as Case 1 , we have in (9) $\gamma=\left(\delta_{0} \delta \varepsilon_{0}^{p^{\kappa}}\right)^{p^{-(n-1)}} p^{n-1}$. Therefore, if we choose $\delta$ so that $\operatorname{Sp}(\gamma)$ $\equiv p^{n-1} \bmod p^{n}$, we have $B \equiv 1 \bmod p^{e_{1} p+(n-1) e-\imath}$ and $(A, B)=\zeta_{n}^{\operatorname{Sp}(\gamma)}=\zeta_{n}^{p^{n-1}} \neq 1$.

Q.E. D.

From Lemma 2, we have following two Lemmas immediately.

LEMMA 3. Let $n \geqq 1$ and $p \neq 2$. Then we have

(i) if $A \sim \pi^{a}, a \in Z$ and ord $a=m(0 \leqq m \leqq n-1)$,

$$
f(A)=e_{1} p+(n-m-1) e+1 .
$$

(ii) if $A=\mathrm{E}\left(\alpha_{\imath}, \pi^{i}\right), \imath \in F, \alpha_{i} \in I$ and ord $\alpha_{\imath}=m(0 \leqq m \leqq n-1)$,

$$
f(A)=e_{1} p+(n-m-1) e-\imath+1 .
$$

Proof. (i) Let $a=a^{\prime} p^{m}\left(a^{\prime} \in \boldsymbol{Z}, a^{\prime} \neq 0 \bmod p\right)$ and $A^{\prime}=\pi^{a^{\prime}}$. Then $K\left(\sqrt[p^{n}]{A}\right)$ $=K\left(\sqrt[p-m]{A^{\prime}}\right)$ and the conductor of $K\left(\sqrt[p-m]{A^{\prime}}\right)$ is $\mathfrak{p}^{e_{1} p+(n-m+1) e+1}$ by Lemma 2 (i) (using $n-m$ instead of $n$ ), so we have $f(A)=e_{1} p+(n-m-1) e+1$. Just as (i) we 
have (ii) from Lemma 2 (ii).

Q.E.D.

LEMMA 4. Let $n \geqq 1$ and $p \neq 2$. Then

(i) $\quad f\left(\pi^{a}\right)>f\left(E\left(\alpha_{\imath}, \pi^{i}\right)\right)$ and $f\left(\pi^{a}\right)>f\left(E^{*}(\alpha)\right)$

where $a \in \boldsymbol{Z}, \alpha_{i} \in I$ ( $i \in F$ ), $0 \leqq$ ord $a \leqq n-1$, ord $a \leqq$ ord $\alpha$ and $\alpha \in I$ is arbitrary.

(ii) $\quad f\left(E\left(\alpha_{\imath}, \pi^{i}\right)\right)>f\left(E\left(\alpha_{\jmath}, \pi^{j}\right)\right)$ and $f\left(E\left(\alpha_{\imath}, \pi^{i}\right)\right)>f\left(E^{*}(\alpha)\right)$

where $i, j \in F(i<j), \alpha_{\imath}, \alpha \in I$ and $0 \leqq$ ord $\alpha_{\imath} \leqq n-1$, ord $\alpha_{\imath} \leqq$ ord $\alpha_{\jmath}$ and $\alpha$ is arbitrary.

Proof. We have the result immediately from Lemma 3 and the fact $E^{*}(\alpha)$ is $p^{n}$-primary.

Now, by local class field theory and by definition of conductor, we have: For elements $B_{1}, \cdots, B_{r}$ of $K$

and

$$
f\left(B_{1} \cdots B_{r}\right) \leqq \operatorname{Max}\left\{f\left(B_{1}\right), \cdots, f\left(B_{r}\right)\right\}
$$

$$
f\left(B_{1} \cdots B_{r}\right)=f\left(B_{1}\right) \quad \text { if } \quad f\left(B_{1}\right)>f\left(B_{\imath}\right) \quad(\imath=2, \cdots, r) .
$$

In fact, by local class field theory and by definition of conductor, the conductor of $L=K\left(\sqrt[p^{n}]{B_{1}}, \cdots, \sqrt[p^{n}]{B_{r}}\right)$ is $p^{\operatorname{Max}\left\{f^{(1)}, \cdots, f^{(r)},\right.}$ where $f^{(i)}=f\left(B_{\imath}\right)(1 \leqq i \leqq r)$. Since $K\left(\sqrt[p^{n}]{B_{1} \cdots B_{r}}\right)$ is a subfield of $L$ we have $f\left(B_{1} \cdots B_{r}\right) \leqq \operatorname{Max}\left\{f^{(1)}, \cdots, f^{(r)}\right\}$.

Next, let $f^{(1)}>f^{(i)}(i=2, \cdots, r)$. Since $K\left(\sqrt[p^{n}]{B_{1} \cdots B_{r}}, \sqrt[p n]{B_{2}}, \cdots, \sqrt[p^{n}]{B_{r}}\right)=L$, we have $\operatorname{Max}\left\{f\left(B_{1} \cdots B_{r}\right), f^{(2)}, \cdots, f^{(r)}\right\}=f^{(1)}$ and it follows that $f\left(B_{1} \cdots B_{r}\right)=f^{(1)}=f\left(B_{1}\right)$.

LEMMA 5. Let $n \geqq 2$ and $p \neq 2$.

If $A_{2} \sim E\left(\alpha_{\imath_{2}}, \pi^{\imath_{2}}\right) \quad\left(i_{2} \in F, \alpha_{\imath_{2}} \in I\right.$, ord $\left.\alpha_{\imath_{2}}=1\right) \quad A_{1} \sim E\left(\alpha_{\imath_{1}}, \pi^{\imath_{1}}\right) \quad\left(\imath_{1} \in F, \alpha_{\imath_{1}} \in I\right.$, ord $\left.\alpha_{\imath_{1}}=0\right)$ and $f_{2}=e_{1} p+(n-2) e-i_{2}+1, \quad f_{1}=e_{1} p+(n-1) e-\imath_{1}+1$ then we have $f\left(A_{2} A_{1}\right) \leqq \operatorname{Max}\left\{f_{2}, f_{1}\right\}$. Moreover, $f\left(A_{2} A_{1}\right)=\operatorname{Max}\left\{f_{2}, f_{1}\right\}$ if and only if $e+\imath_{2} \neq \imath_{1}$ or $\alpha_{\imath_{2}} \varepsilon \neq \alpha_{\imath_{1}} p \bmod p^{2}$ and $e+i_{2}=i_{1}$.

Proof. By Lemma 3(ii), $f\left(A_{2}\right)=f_{2}$ and $f\left(A_{1}\right)=f_{1}$. By (15) we have $f \leqq \operatorname{Max}\left\{f_{2}, f_{1}\right\}$ where $f=f\left(A_{2} A_{1}\right)$. And if $f_{2} \neq f_{1}$ (i. e. $\left.e+i_{2} \neq i_{1}\right)$ then $f=\operatorname{Max}\left\{f_{2}, f_{1}\right\}$ by (15).

Next, we show that if $e+i_{2}=i_{1}$ (i.e. $f_{2}=f_{1}$ ) and $\alpha_{\imath_{2}} \varepsilon \neq \alpha_{\imath_{1}} p \bmod p^{2}$ then $f=f_{2}=f_{1}$.

Since $f \leqq f_{2}=f_{1}$ it is enough to show that there exists $B$ such that $B \equiv 1$ $\bmod \mathfrak{p}^{f_{2}-1}$ and $\left(A_{2} A_{1}, B\right) \neq 1$.

Since $e+i_{2}=i_{1}$ and $i_{2}, i_{1} \in F$ it follows that $e_{1}>i_{2} \geqq 1$. Let $\jmath_{2}=e_{1} p-\imath_{2}$ then $j_{2} \in F$ and $j_{2}>e_{1}$.

By the assumption $\alpha_{\imath_{2}} \varepsilon \neq \equiv \alpha_{\imath_{1}} p \bmod p^{2}$, there exists $\delta_{0}\left(\delta_{0} \in R^{\times}\right)$such that $j_{2}\left(\alpha_{\imath_{2}}-\alpha_{\imath_{1}} \varepsilon^{-1} p\right) \equiv \delta_{0} p \bmod p^{2}$ and for this $\delta_{0}$ we choose $\delta\left(\delta \in R^{\times}\right)$satisfying $\operatorname{Sp}\left(\left(\delta_{0} \delta \varepsilon_{0}^{p \kappa}\right)^{p^{-(n-1)}}\right) \equiv 1 \bmod p$. Now, let $B=E\left(\beta_{\jmath_{\varepsilon}}, \pi^{\jmath_{2}}\right)$ where $\beta_{\jmath_{2}}=\delta p^{n-2}$ then $B \equiv 1 \bmod \mathfrak{p}^{f_{2}-1}$.

And,

$$
E\left(j_{2} \alpha_{\imath_{2}} \beta_{\jmath_{2}}, \pi^{\imath_{1}+\jmath_{2}}\right) \equiv 1-j_{2} \alpha_{\imath_{2}} \delta p^{n-2} \pi^{e_{1} p} \quad \bmod \mathfrak{p}^{e_{1} p+(n-1) e+1}
$$


Thus,

$$
E\left(j_{2} \alpha_{\imath_{1}} \beta_{\jmath_{2}}, \pi^{\imath_{1}+\jmath_{2}}\right) \equiv 1-j_{2} \alpha_{\imath_{1}} \delta p^{n-2} \pi^{e_{1} p+e} \quad \bmod \mathfrak{p}^{e_{1} p+(n-1) e+1}
$$

$$
\begin{gathered}
E\left(j_{2} \alpha_{\imath_{2}} \beta_{\jmath_{2}}, \pi^{\imath_{2}+\jmath_{2}}\right) E\left(j_{2} \alpha_{\imath_{1}} \beta_{\jmath_{2}}, \pi^{\imath_{1}+\jmath_{2}}\right) \equiv 1-\jmath_{2}\left(\alpha_{\imath_{2}}-\alpha_{\imath_{1}} \varepsilon^{-1} p\right) \delta p^{n-2} \pi^{e_{1} p} \\
\equiv 1-\delta_{0} \delta \varepsilon_{0}^{p \kappa} p^{n-1} \pi_{1}^{p} \quad \bmod p^{e_{1} p+(n-1) e+1}
\end{gathered}
$$

On the other hand, by (7),

$$
E^{*}\left(\left(\delta_{0} \delta \varepsilon_{0}^{p^{\kappa}}\right)^{p^{-(n-1)}} p^{n-1}\right) \equiv 1-\delta_{0} \delta \varepsilon_{0}^{p^{\kappa}} p^{n-1} \pi_{1}^{p} \quad \bmod \mathfrak{p}^{e_{1} p+(n-1) e+1} .
$$

So we have, in explicit formula (9), $\gamma=\left(\delta_{0} \delta \varepsilon_{0}^{p^{\kappa}}\right)^{p^{-(n-1)}} p^{n-1}$ where

$$
E\left(j_{2} \alpha_{\imath_{2}} \beta_{\jmath_{2}}, \pi^{\imath_{2}+\jmath_{2}}\right) E\left(j_{2} \alpha_{\imath_{1}} \beta_{\jmath_{2}}, \pi^{\imath_{1}+\jmath_{2}}\right) \sim \cdots E^{*}(\gamma) .
$$

And $\operatorname{Sp}(\gamma) \equiv \operatorname{Sp}\left(\left(\delta_{0} \delta \varepsilon_{0}^{p^{\kappa}}\right)^{p^{-(n-1)}} p^{n-1}\right) \equiv p^{n-1} \bmod p^{n}$, so we have $\left(A_{2} A_{1}, B\right)=\zeta_{n}^{\mathrm{Sp}(\gamma)}$ $=\zeta_{n}^{p-1} \neq 1$.

Finally, we show that if $e+i_{2}=i_{1}$ and $\alpha_{\imath_{2}} \varepsilon \equiv \alpha_{\imath_{1}} p \bmod p^{2}$ then we have $f \leqq f_{2}-1$.

Now, let $n \geqq 2$ and (̌̌ $) \quad B=\prod_{\jmath} E\left(\beta_{\jmath}, \pi^{j}\right) E^{*}(\beta) \equiv 1 \bmod \mathfrak{p}^{f_{2}-1}$ then we have $\beta_{j} \equiv 0$ $\bmod p^{n-2}$ for all $j \in F$ and

$$
\beta_{j} \equiv \begin{cases}0 \bmod p^{n-1} & \text { if } \quad j<e_{1} p-i_{2} \\ 0 \bmod p^{n} & \text { if } j \leqq e_{1} p-e-i_{2} .\end{cases}
$$

The proof is quite similar to that of Lemma 1.

Therefore,

$$
\prod_{\jmath} E\left(j \alpha_{\imath_{2}} \beta_{\jmath}, \pi^{\imath_{2}+j}\right) E\left(j \alpha_{\imath_{1}} \beta_{\jmath}, \pi^{\imath_{1}+j}\right) \sim E\left(j_{2} \alpha_{\imath_{2}} \beta_{\jmath_{2}}, \pi^{\imath_{2}+\jmath_{2}}\right) E\left(j_{2} \alpha_{\imath_{1}} \beta_{\jmath_{2}}, \pi^{\imath_{1}+\jmath_{2}}\right),
$$

where $j_{2}=e_{1} p-i_{2}$, i.e. if $j \neq j_{2}, E\left(j \alpha_{\imath_{2}} \beta_{\jmath}, \pi^{\imath^{2+j}}\right) \sim 1$ and $E\left(j \alpha_{\imath_{1}} \beta_{\jmath}, \pi^{\imath_{1}+\jmath}\right) \sim 1$. In fact, if $j<e_{1} p-i_{2}$ then $\alpha_{\imath_{2}} \beta_{j} \equiv 0 \bmod p^{n}$, if $j>e_{1} p-i_{2}$ then $\alpha_{\imath_{2}} \beta_{j} \equiv 0 \bmod p^{n-1}$ and $\operatorname{ord}^{\times} E\left(j \alpha_{\imath_{2}} \beta_{j}, \pi^{\imath_{2}+j}\right)>e_{1} p+(n-1) e$. And if $j \leqq e_{1} p-e-\imath_{2}$ then $\alpha_{\imath_{1}} \beta_{j} \equiv 0 \bmod p^{n}$, if $e_{1} p-e-i_{2}<j<e_{1} p-i_{2}$ then $\alpha_{\imath_{1}} \beta_{j} \equiv 0 \bmod p^{n-1}$ and $\operatorname{ord}^{\times} E\left(j \alpha_{\imath_{1}} \beta_{j}, \pi^{\imath_{1}+j}\right)$ $>e_{1} p-e-i_{2}+i_{1}+(n-1) e=e_{1} p+(n-1) e$, because $e+i_{2}=\imath_{1}$. And if $e_{1} p-\imath_{2}<\jmath$ then $\alpha_{\imath_{1}} \beta_{j} \equiv 0 \bmod p^{n-2}$ and

Now,

$$
\operatorname{ord}^{\times} E\left(j \alpha_{\imath_{1}} \beta_{\jmath}, \pi^{\imath_{1}+j}\right)>i_{1}+e_{1} p-\imath_{2}+(n-2) e=e_{1} p+(n-1) e .
$$

$$
\begin{gathered}
E\left(\jmath_{2} \alpha_{\imath_{2}} \beta_{\jmath_{2}}, \pi^{\imath_{2}+\jmath_{2}}\right) E\left(j_{2} \alpha_{\imath_{1}} \beta_{\jmath_{2}}, \pi^{\imath_{1}+\jmath_{2}}\right) \equiv\left(1-\jmath_{2} \alpha_{\imath_{2}} \beta_{\jmath_{2}} \pi^{e_{1} p}\right)\left(1-\jmath_{2} \alpha_{\imath_{1}} \beta_{\jmath_{2}} \pi^{e_{1} p+e}\right) \\
=1-j_{2}\left(\alpha_{\imath_{2}}-\varepsilon^{-1} \alpha_{\imath_{1}} p\right) \beta_{\jmath_{2}} \pi^{e_{1} p} \quad \bmod p^{e_{1} p+(n-1) e+1} .
\end{gathered}
$$

While by the assumption $\alpha_{\imath_{2}}-\varepsilon^{-1} \alpha_{\imath_{1}} p \equiv 0 \bmod p^{2}$ and $\beta_{\jmath_{2}} \equiv 0 \bmod p^{n-2}$ so we have $E\left(j_{2} \alpha_{\imath_{2}} \beta_{\jmath_{2}}, \pi^{\imath_{2}+\jmath_{2}}\right) E\left(j_{2} \alpha_{\imath_{1}} \beta_{\jmath_{2}}, \pi^{\imath_{1}+\jmath_{2}}\right) \sim 1$ by (12). Consequently $\gamma \equiv 0 \bmod p^{n}$, $\mathfrak{P}$ in (9).

Thus, we have shown $\left(A_{2} A_{1}, B\right)=\zeta_{n}^{\mathrm{Sp}(r)}=1$ for any $B$, such that $B \equiv 1$ $\bmod \mathfrak{p}^{f_{2}-1}$.

Q. E. D. 
Now, we prove Theorem 1 in the case $p \neq 2$.

Let $A_{\widetilde{p}^{n}} \pi^{a} \prod_{\imath} E\left(\alpha_{\imath}, \pi^{i}\right) E^{*}(\alpha)$ and $f=f(A)$. When $n=1$ and $i_{1}=0$ by Lemma 2 , Lemma 4 (i) and (15), we have $f=f\left(\pi^{a}\right)=e_{1} p+1=f_{1}$.

If $i_{1} \geqq 1, A_{\widetilde{p}} \prod_{i \geqq \imath_{1}} E\left(\alpha_{\imath}, \pi^{i}\right) E^{*}(\alpha)$ and by Lemma 4 and (15) $f=f\left(E\left(\alpha_{\imath_{1}}, \pi^{\imath_{1}}\right)\right)$ $=e_{1} p-i_{1}+1=f_{1}$ and $f_{1}=\operatorname{Max}\left\{f_{1}, f_{2}\right\}$ because $f_{2}<f_{1}$ by the definition $i_{n+1}=i_{n}$. Next let $n \geqq 2$, if $0=i_{2}=i_{1}$ we have $f=e_{1} p+(n-1) e+1=f_{1}$ by Lemma 2 , Lemma 4 and (15), and $f_{1}=\operatorname{Max}\left\{f_{2}, f_{1}\right\}$ because $i_{2}=i_{1}$. If $0=i_{2}<i_{1}$ then $A=A_{2} A_{1}$ where

$$
A_{2}=\left\{\begin{array}{l}
\pi^{a}: i_{1}=1 \quad(\text { ord } a=1) \\
\pi^{a} \prod_{\imath<i_{1}} E\left(\alpha_{\imath}, \pi^{i}\right): i_{1}>1 \quad\left(\text { ord } a=\operatorname{ord} \alpha_{\imath}=1\right) .
\end{array}\right.
$$

and

$$
A_{1}=\prod_{i \geqq \imath_{1}} E\left(\alpha_{\imath}, \pi^{i}\right) E^{*}(\alpha) \quad\left(0=\operatorname{ord} \alpha_{\imath_{1}} \leqq \text { ord } \alpha_{\imath}\right) .
$$

Thus we have $f\left(A_{2}\right)=e_{1} p+(n-2) e+1=f_{2}$ by Lemma 3 , Lemma 4 and (15), $f\left(A_{1}\right)=e_{1} p+(n-1) e-i_{1}+1=f_{1}$ by Lemma 2 , Lemma 4 and (15).

Since $e+i_{2} \neq i_{1}, f_{1} \neq f_{2}$ and we have $f=\operatorname{Max}\left\{f_{2}, f_{1}\right\}$ by (15).

If $1 \leqq i_{2}<i_{1}$ then $A=A_{3} A_{2} A_{1}$ where

$$
\begin{aligned}
& A_{3}=\left\{\begin{array}{l}
\pi^{a}: i_{2}=1 \quad(\text { ord } a \geqq 2) \\
\pi^{a} \prod_{\imath<i_{2}} E\left(\alpha_{\imath}, \pi^{i}\right): i_{2}>1 \quad\left(\text { ord } \alpha_{i} \geqq 2\right),
\end{array}\right. \\
& A_{2}=\prod_{\imath_{2} \leqq \imath<i_{1}} E\left(\alpha_{\imath}, \pi^{i}\right) \quad\left(\text { ord } \alpha_{i}=1\right)
\end{aligned}
$$

and

$$
A_{1}=\prod_{i \geqq \imath_{1}} E\left(\alpha_{\imath}, \pi^{i}\right) E^{*}(\alpha) \quad\left(0=\operatorname{ord} \alpha_{\imath_{1}} \leqq \operatorname{ord} \alpha_{\imath}\right) .
$$

Now, since ord $a \geqq 2$ and ord $\alpha_{i} \geqq 2 \quad\left(\imath<\imath_{2}\right)$ we have $f\left(A_{3}\right) \leqq e_{1} p+(n-3) e+1$ by Lemma 3 and (15) and $e_{1} p+(n-3) e+1<\operatorname{Max}\left\{f_{2}, f_{1}\right\}$ because

$$
f_{1}-\left(e_{1} p+(n-3) e+1\right)=2 e-\imath_{1} \geqq 2 e-\left(e_{1} p-1\right)>0 .
$$

And $f\left(A_{2}\right)=f_{2}, \quad f\left(A_{1}\right)=f_{1}$ by Lemma 4 and (15). Therefore $f=f\left(A_{3} A_{2} A_{1}\right)$ $\leqq \operatorname{Max}\left\{f_{2}, f_{1}\right\}$ by (15). Moreover if $e+i_{2} \neq i_{1}$ or if $e+i_{2}=i_{1}$ and $\alpha_{\imath_{2}} \varepsilon \neq \alpha_{\imath_{1}} p \bmod p^{2}$, then $A_{2} A_{1}=E\left(\alpha_{\imath_{2}}, \pi^{\imath_{2}}\right) E\left(\alpha_{\imath_{1}}, \pi^{\imath_{1}}\right) B$ where

$$
B=\prod_{\substack{i>2_{2} \\ \imath \neq i_{1}}} E\left(\alpha_{\imath}, \pi^{i}\right) E^{*}(\alpha) .
$$

By Lemma $5 f\left(E\left(\alpha_{\imath_{2}}, \pi^{\imath_{2}}\right) E\left(\alpha_{\imath_{1}}, \pi^{\imath_{1}}\right)\right)=\operatorname{Max}\left\{f_{2}, f_{1}\right\}$ and $f(B)<\operatorname{Max}\left\{f_{2}, f_{1}\right\}$ by Lemma 4 and (15), so we have $f\left(A_{2} A_{1}\right)=\operatorname{Max}\left\{f_{2}, f_{1}\right\}$ and $f=f\left(A_{3} A_{2} A_{1}\right)=\operatorname{Max}\left\{f_{2}, f_{1}\right\}$. If $e+i_{2}=i_{1}$ and $\alpha_{\imath_{2}} \varepsilon \equiv \alpha_{\imath_{1}} p \bmod p^{2}$ then $f\left(E\left(\alpha_{\imath_{2}}, \pi^{\imath_{2}}\right) E\left(\alpha_{\imath_{1}}, \pi^{\imath_{1}}\right)\right)<\operatorname{Max}\left\{f_{2}, f_{1}\right\}$ by Lemma 5 , and $f=f\left(A_{3} A_{2} A_{1}\right)<\operatorname{Max}\left\{f_{2}, f_{1}\right\}$ from (15).

Finally, in the case $1 \leqq i_{2}=\imath_{1}, A=A_{3} A_{1}$ where 


$$
A_{3}=\left\{\begin{array}{l}
\pi^{a}: i_{1}=1 \quad(\text { ord } a \geqq 2) \\
\pi^{a} \prod_{\imath<i_{1}} E\left(\alpha_{\imath}, \pi^{i}\right): i_{1}>1 \quad\left(\text { ord } a \geqq 2, \text { ord } \alpha_{i} \geqq 2\right)
\end{array}\right.
$$

and

$$
A_{1}=\prod_{i \geqq \imath_{1}} E\left(\alpha_{\imath}, \pi^{i}\right) E^{*}(\alpha) \quad\left(\operatorname{ord} \alpha_{i} \geqq \operatorname{ord} \alpha_{\imath_{1}}=0\right) .
$$

Just as before, we have $f=f_{1}$ because $f\left(A_{3}\right) \leqq e_{1} p+(n-3) e+1<f_{1}=f\left(A_{1}\right)$, and $f_{1}=\operatorname{Max}\left\{f_{2}, f_{1}\right\}$ because $i_{2}=i_{1}$.

Thus the proof of Theorem 1 in the case $p \neq 2$ is completed.

\section{PROOF OF THEOREM 1 IN THE CASE $p=2$.}

The difference with the case $p \neq 2$ is that, in the explicit formula (10) another term $\prod_{\mu, \nu=1}^{\infty} E\left(\left(2^{\mu-1} \imath+2^{\nu-1} j\right) \alpha_{\imath}^{F^{\mu}} \beta_{\jmath}^{P^{\nu}}, \pi^{\left.2^{\mu_{2+2^{\nu} j}}\right)}\right.$ is multiplied to each $E\left(j \alpha_{i} \beta_{\jmath}, \pi^{\imath+j}\right)$. But for all $\alpha_{\imath}, \beta$, which appear in the proofs of Lemma 2 and Lemma 5 in the case $p \neq 2, \gamma_{\imath \jmath \mu \nu} \equiv 0 \bmod p^{n}, \mathfrak{P}$ for all $\mu, \nu(\mu \geqq 1, \nu \geqq 1)$ where

$$
E\left(\left(2^{\mu-1} i+2^{\nu-1} j\right) \alpha_{\imath}^{P^{\mu}} \beta_{\jmath}^{P^{\nu}}, \pi^{2^{\mu_{\imath+2^{\nu} j}}}\right) \sim \cdots E^{*}\left(\gamma_{\imath \jmath \mu \nu}\right)
$$

Therefore the multiplied term gives no influence to the class of $\gamma \bmod p^{n}, \mathfrak{P}$. Thus, having Lemma 3, 4 which are corollaries of Lemma 2, Theorem 1 holds also for $p=2$.

\section{$\S 4$. Remarks and examples.}

Remark 1. By elementary but rather complicated calculations of the explicit formula we can prove Theorem 1 without (15).

Remark 2. Let $n=1$ and $A_{\sim} \prod_{\imath} E\left(\alpha_{\imath}, \pi^{i}\right) E^{*}(\alpha)$ then Theorem 1 asserts that the conductor of $K(\sqrt[p]{\bar{A}}) / K$ is $\mathfrak{p}^{e_{1} p-\imath_{1}+1}$. On the other hand, the number $i_{1}$ is characterized by the following congruences:

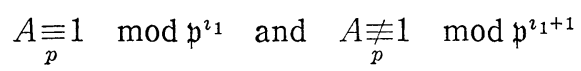

where, generally, the notation $A \underset{p^{m}}{\equiv} 1 \bmod \mathfrak{p}^{k}(m \geqq 1, k \geqq 1)$ means that there exists a principal unit $\eta$ of $K$ such that $A \eta^{-p^{m}} \equiv 1 \bmod p^{k}$. This result is known $(\mathrm{H}$. Hasse [1], $\mathrm{I}_{a}$, p. 90, Satz. 10). While, when $n \geqq 2$ it is impossible in general to determine the conductor of $K\left(\sqrt[p^{n}]{A}\right) / K$ by analogous congruences.

For example, let $K=\boldsymbol{Q}_{p}\left(\zeta_{2}\right)(p \neq 2)$ and

$$
A_{p^{2}} E\left(\alpha_{\imath_{2}}, \pi^{\imath_{2}}\right) E\left(\alpha_{\imath_{1}}, \pi^{\imath_{1}}\right)
$$

where

$$
\text { ord } \alpha_{\imath_{2}}=1 \quad\left(2 \leqq i_{2} \leqq e_{i}-1=p-1\right)
$$


and

$$
\text { ord } \alpha_{\imath_{1}}=0 \quad\left(\imath_{1}=e+1=p(p-1)+1\right) .
$$

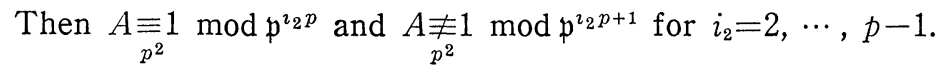

While, since $f_{1}=e_{1} p>f_{2}=e_{1} p-i_{2}+1$ for any $\imath_{2}\left(2 \leqq i_{2} \leqq p-1\right)$, the conductor of $K(\sqrt[p]{A}) / K$ is $\mathfrak{p}^{e_{1} p}$ by Theorem 1 .

Example 1. Let $K \ni \zeta_{n}$ and $\pi$ be a prime of $K$.

(i) Let $A=\pi^{a} \eta$ where $a \in \boldsymbol{Z}, a \neq 0 \bmod p$ and $\eta$ is a unit of $K$, then the conductor of $K\left(\sqrt[p^{n}]{A}\right) / K$ is $\mathfrak{p}^{e_{1} p+(n-1) e+1}$.

For, since $i_{1}=0$ we have $f=\operatorname{Max}\left\{f_{1}, f_{2}\right\}=f_{1}=e_{1} p+(n-1) e+1$ by Theorm 1 .

(ii) Let $n \geqq 2$ and $A=\pi^{p}\left(1-\pi^{j}\right)\left(e<j<e_{1} p\right)$, then the conductor of $K\left(\sqrt[p^{n}]{A}\right) / K$ is $\mathfrak{p}^{e_{1} p+(n-2) e+1}$.

For, since $i_{2}=0$ and $i_{1}=\jmath$ we have $e+\imath_{2}<\imath_{1}$ and $f=\operatorname{Max}\left\{f_{2}, f_{1}\right\}=f_{2}=$ $e_{1} p+(n-2) e+1$.

Example 2. Let $K=\boldsymbol{Q}_{p}\left(\boldsymbol{\zeta}_{n}\right)$ then the conductor of $K\left(\sqrt[p^{n}]{\zeta_{m}}\right) / K(1 \leqq m \leqq n)$ is $\mathfrak{p}^{e_{1} p+(m-1) e}$.

For, let $1-\pi=\zeta_{n}=\prod_{\imath} E\left(\alpha_{\imath}, \pi^{i}\right) E^{*}(\alpha)\left(\alpha_{1} \neq 0 \bmod p\right)$ then

$$
\zeta_{m}=\zeta_{n}^{p^{n-m}}=\prod_{\imath} E\left(\alpha_{\imath} p^{n-m}, \pi^{i}\right) E\left(\alpha p^{n-m}\right) .
$$

Therefore, since $i_{n-m}$ does not exist and $i_{n-m+1}=1$, we have $f=f_{n-m+1}=e_{1} p+(m-1) e$ by Theorem 2 .

Example 3. For some Kummer extensions we can get the ramification subgroups from conductors obtained by Theorem 1 . For example, let $K \ni \zeta_{n}(n \geqq 1)$ and $L=K\left(\sqrt[p^{n}]{A_{i}^{\alpha_{i}}}\right)$ where $i=0$ or $i \in F$ and $A_{0}=\pi^{\alpha_{0}} \quad\left(\alpha_{0} \in Z, \quad \alpha_{0} \neq 0 \quad \bmod p\right)$, $A_{\imath}=E\left(\alpha_{\imath}, \pi^{i}\right)\left(\imath \in F, \alpha_{i} \in I, \alpha_{i} \neq 0 \bmod p\right)$. Now, let $G=\langle\sigma\rangle=\operatorname{Gal}(L / K)$ and $G$, be the $\jmath$-th ramification subgroup of this extension:

$$
\begin{aligned}
G=G_{0}=\cdots=G_{m_{1}}=\langle\sigma\rangle \supseteqq G_{m_{1}+1}=\cdots & =G_{m_{2}}=\left\langle\sigma^{p}\right\rangle \supsetneq \cdots \\
& =G_{m_{n}}=\left\langle\sigma^{p^{n-1}}\right\rangle \supsetneq G_{m_{n+1}}=\{1\} .
\end{aligned}
$$

Then, we have $m_{k}=e_{1} p^{k}-\imath$ for $k=1,2, \cdots, n$.

Proof. Since $L / K$ is a totally ramified cyclic extension of degree $p^{n}$, we only need to calculate $m_{k}$. Now, by Theorem 1 (or by Lemma 2) we have $f^{(s)}=e_{1} p+(s-1) e-i+1(1 \leqq s \leqq n)$ where $\mathfrak{p}^{f^{(s)}}$ is the conductor of $K\left(\sqrt[p^{s}]{A_{2}^{\alpha_{i}}}\right)$. Thus,

$$
\begin{array}{ll}
f^{(1)}=e_{1} p-i+1=\frac{1}{\# G_{0}} \sum_{j=0}^{m_{1}} \sharp G_{j}=m_{1}+1 \quad \text { and so } m_{1}=e_{1} p-i . \\
f^{(2)}=\frac{1}{\# G_{0}} \sum_{j=0}^{m_{2}} \sharp G_{j}=f^{(1)}+\left(m_{2}-m_{1}\right) p^{-1} \quad \text { and so } m_{2}=e_{1} p+m_{1},
\end{array}
$$


because $f^{(2)}-f^{(1)}=e$. By repeating this process, we have

$$
m_{k}=e p^{k-1}+e p^{k-2}+\cdots+e p+m_{1}=e_{1} p^{k}-i,
$$

because $e_{1}(p-1)=e$.

Q.E.D.

\section{REFERENCES}

[1] H. HASSE, Bericht über neuere Untersuchungen und Probleme aus der Theorie der algebraishen Zahlenkörper, Physica-Verlag (1970).

[2] H. Hasse, Zahlentheorie, Akademie-Verlag (1969).

[3] H. HASSE, Zur Arbeit von I. R. ŠAFAREVIČ über das allgemeine Reziprozitätsgesetz, Math. Nach. 5 (1951), 301-327.

[4] M. KNeser, Zum expliziten Reziprozitätsgesetz von I.R. ŠAFAREVIČ, Math. Nach. 6 (1951), 89-96.

[5] I. R. ŠAFAREvič, A general reciprocity law, J. Math. Sbornik 26 (1950), 113-146.

[6] J.P. Serre, Corps locaux, Hermann (1962).

Department of Mathematics,

MiYagi University of Education,

SENDAI, JAPAN 